\title{
Autophagy regulation: RNF2 targets AMBRA1
}

\author{
Cell Research (2014) 24:1029-1030. doi:10.1038/cr.2014.105; published online 8 August 2014
}

The phosphatidylinositol 3-kinase complex I (PI3K complex I) is a crucial regulator of autophagy, which contains Beclin 1 (or ATG6), ATG14L, VPS34 (or the class III phosphatidylinositol 3-kinase and its adaptor VPS15) and AMBRA1, and controls autophagosome formation. In a paper recently published in Cell Research, Xia et al. report that during nutrient deprivation the ubiquitin E3 ligase RNF2 is recruited to the PI3K complex I, and ubiquitinates AMBRA1 to trigger its degradation and downregulate autophagy.

Macroautophagy (hereafter called autophagy) is a lysosomal degradation pathway for cytoplasmic components [1]. The ubiquitin-proteasome pathway is also critical during the process of autophagy. The formation of autophagosomes (double-membrane bound vacuoles that sequester cargo in bulk or in a selective manner before their delivery to the lysosomal compartment) depends on ubiquitination-like activity [2]. The selective removal of cargo (e.g., protein aggregates, organelles) by autophagy is dependent in many cases on the ubiquitination of the cargo [3]. Recent studies also indicate that ubiquitination regulates the activity of autophagy proteins that comprise autophagosomes [4].

Autophagosome formation is dependent on evolutionarily conserved ATG (autophagy-related) proteins initially identified in yeast [2]. These proteins function in complexes or functional modules on a membrane known as the phagophore that matures into the autophagosome via stages of initiation, elongation, and sealing. Phosphati- dylinositol 3-phosphate kinase complex I (PI3K complex I) plays a key role in the initiation step. In this complex, Beclin 1 (the mammalian homolog of yeast ATG6) interacts with ATG14L, AMBRA1, and class III PI3K or VPS34 [2] (Figure 1). The activity of this complex, which produces the lipid phosphatidylinositol 3-phosphate (PI3P), is crucial to recruitment of ATG, which is required for the elongation and sealing of the autophagosomal membrane.

Recently, Xia and colleagues [5] demonstrate that RNF2 (also called Ring1B) regulates autophagosome formation in response to nutrient starvation by influencing the ubiquitination of AMBRA1. RNF2 is a member of the RING-domain ubiquitin E3 ligase family [6]. In a series of experiments involving two-hybrid screens with RNF2 as bait, Xia and colleagues [5] showed that RNF2 interacts with AMBRA1 and that this interaction is enhanced upon autophagy stimulation in cells cultured in the absence of serum and amino acids. Deletion of $R N F 2$ robustly stimulates autophagy in response to starvation whereas restoration of RNF2 in $\mathrm{RNF}^{-/-}$mouse embryo fibroblasts (MEFs) reduces autophagy. An RNF2 mutant with no E3 ligase activity does not impair autophagy in RNF2 $2^{--}$MEFs. The authors further showed that RNF2 downregulates autophagy by promoting the degradation of AMBRA1. RNF2 catalyzes the K48-linked polyubiquitination of AMBRA1 which mediates its proteasomal degradation. The crucial site for AMBRA1 K48-linked polyubiquitination is lysine 45 , and a K45R AMBRA1 mutant is not sensitive to RNF2-mediated ubiquitination and is able to sustain VPS34 activity and autophagy. Beclin 1 exists in different complexes involved in different steps of the autophagic pathway [2]. In the current study, the authors showed that RNF2 is associated with the PI3K complex 1 with Beclin 1 ATG14 and AMBRA1, a complex involved in early stage of autophagosome formation. Interestingly, in the absence of RNF2, the association of Beclin 1 with VPS34 in the PI3K complex 1 is enhanced.

Recently, Fan's group reported that AMBRA1-DDB1-CUL4A is the E3 ligase that mediates K63-linked ubiquitination of Beclin 1 to enhance its binding to VPS34 in response to starvation [7]. In the present study [5], the group showed that the K63-linked ubiquitination of Beclin 1 is inhibited by RNF2. A screen identified WASH as an interactor of RNF2. WASH is part of a complex that promotes actin polymerization to facilitate endosomal protein sorting [8] and has been recently shown to interact with Beclin 1 and to be associated with autophagosomal membrane [7]. This interaction impairs the AMBRA1-mediated $\mathrm{K} 63$ polyubiquitination of Beclin 1. WASH recruits RNF2 to AMBRA1 and the PI3K complex 1 in response to starvation. The absence of WASH abolishes the K48-linked polyubiquitination of AMBRA1. In addition, WASH overexpression partially impairs the interaction of AMBRA1 with Beclin1 to block its K63-linked polyubiquitination [7]. The study by Xia et al. reveals a novel layer of regulation: WASH recruits RNF2 to promote AMBRA1 degradation to impair Beclin 1 ubiquitination [5]. This work points to the complex role of ubiquitination in the regulation 


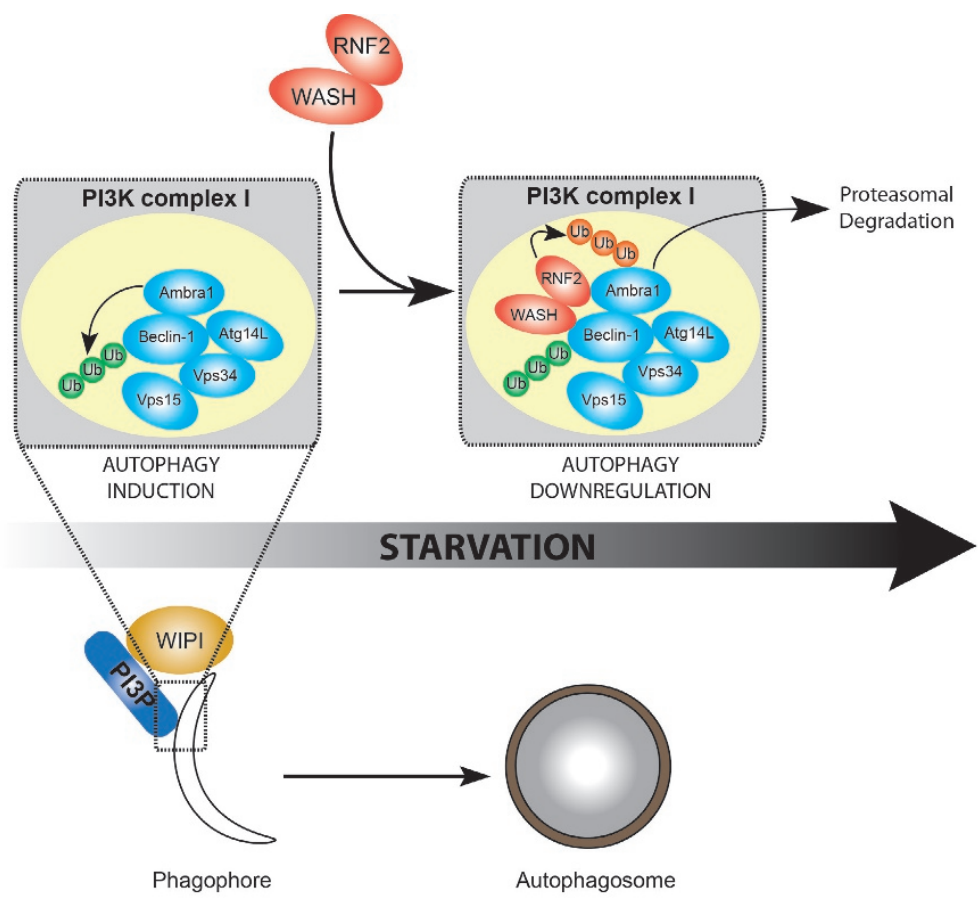

Figure 1 Role of RNF2 and WASH during autophagosome formation. Upon autophagy stimulation by nutrient deprivation, Beclin 1 forms a complex with VPS34 and its adaptor VPS15, ATG14L, and AMBRA1. In this complex, Beclin 1 is K63-polyubiquitylated (green circles) by the E3 ligase AMBRA1 to activate the production of PI3P by VPS34. The production of PI3P at the phagophore recruits WIPI proteins to trigger the ATG machinery to elongate and seal the membrane to form an autophagosome. After the induction of autophagy, WASH and RNF2 are recruited to downregulate the autophagy pathway by inhibiting the VPS34 activity. WASH negatively regulates autophagy through suppression of Beclin $1 \mathrm{~K} 63$-linked polyubiquitination whereas RNF2 is recruited to the complex via the Beclin 1 interactor WASH. RNF2 catalyzes K48-linked polyubiquitination (orange circles) of AMBRA1 to mediate its proteasomal degradation.

of the early stage of autophagy with a balance between activating K63-linked polyubiquitination of Beclin 1 and inhibitory K48-linked polyubiquitination of AMBRA1. These post-translational modifications depend on the activity of two E3 ligases, AMBRA1 and RNF2. This study also stresses the importance of AMBRA1 in stabilization of proteins engaged in the early stage of autophago- some formation via its E3 ligase activity with DDB1-CUL4A to enhance Beclin 1 association with VPS34 [7] and with TRAF6 to stabilize ULK1, which acts in a complex upstream of the PI3K complex 1 in autophagy [9]. Finally, the study by Xia et al. emphasizes that autophagy must be tightly regulated to avoid deleterious effects on cell homeostasis.
The present study raises several questions regarding how and when WASH and RNF2 are recruited to downregulate autophagy in response to starvation. Recently it has been shown that WASH is a positive modulator of autophagosome biogenesis in mammalian cells through regulation of endosomal trafficking of ATG9A [10]. Altogether, these findings suggest that the role of WASH in autophagy is dependent on its subcellular localization and its partners in intracellular membranes.

Etienne Morel $^{1}$, Nicolas Dupont ${ }^{1}$, Patrice Codogno ${ }^{1}$

${ }^{1}$ INSERM U1151-CNRS UMR 8253, Institut Necker Enfants-Malades (INEM) Université, Paris Descartes-Sorbonne Paris Cité Paris, 75993 Paris, cedex 14, France

Correspondence: Etienne Morel ${ }^{\mathrm{a}}$, Patrice Codogno $^{\mathrm{b}}$

aE-mail: etienne.morel@inserm.fr

bE-mail: patrice.codogno@inserm.fr

\section{References}

1 Boya P, Reggiori F, Codogno P. Nat Cell Biol 2013; 15:713-720.

2 Mizushima N, Yoshimori T, Ohsumi Y. Annu Rev Cell Dev Biol 2011; 27:107-132.

3 Stolz A, Ernst A, Dikic I. Nat Cell Biol 2014; 16:495-501.

4 Kuang E, Qi J, Ronai Z. Trends Biochem Sci 2013; 38:453-460.

5 Xia P, Wang S, Huang G, et al. Cell Res 2014; 24:943-958.

6 Voncken JW, Roelen BA, Roefs M, et al. Proc Natl Acad Sci USA 2003; 100:24682473.

7 Xia P, Wang S, Du Y, et al. EMBO J 2013; 32:2685-2696.

8 Seaman MN, Gautreau A, Billadeau DD. Trends Cell Biol 2013; 23:522-528.

9 Nazio F, Strappazzon F, Antonioli M, et al. Nat Cell Biol 2013; 15:406-416.

10 Zavodszky E, Seaman MN, Moreau K, et al. Nat Commun 2014; 5:3828. 